\title{
On the Capacity of Decode-and-Forward Relaying over Rician Fading Channels
}

\author{
Manav R. Bhatnagar, Senior Member, IEEE
}

\begin{abstract}
In this letter, we derive the probability density function (PDF) and cumulative distribution function (CDF) of the minimum of two non-central Chi-square random variables with two degrees of freedom in terms of power series. With the help of the derived PDF and CDF, we obtain the exact ergodic capacity of the following adaptive protocols in a decode-and-forward (DF) cooperative system over dissimilar Rician fading channels: (i) constant power with optimal rate adaptation; (ii) optimal simultaneous power and rate adaptation; (iii) channel inversion with fixed rate. By using the analytical expressions of the capacity, it is observed that the optimal power and rate adaptation provides better capacity than the optimal rate adaptation with constant power from low to moderate signal-to-noise ratio values over dissimilar Rician fading channels. Despite low complexity, the channel inversion based adaptive transmission is shown to suffer from significant loss in capacity as compared to the other adaptive transmission based techniques over DF Rician channels.
\end{abstract}

\section{INTRODUCTION}

Information theoretic study of a cooperative network is a useful tool for implementation of this technology in current and future wireless networks. There exists many useful works over the capacity analysis of the cooperative networks [1] [4]. The outage performance of the cooperative system with Rayleigh fading in the low signal-to-noise ratio (SNR) regime is studied in [1]. Closed-form expressions of the outage probability over Rayleigh and Nakagami- $m$ fading channels are derived in [2] and [3], respectively, with decode-andforward (DF) relays. The outage capacity performance of the two hop based amplify-and-forward (AF) protocol over dissimilar Rayleigh fading channels is studied in [4].

All the aforementioned works only consider fixed rate and fixed power transmission based schemes. However, if the source/relay terminal possesses the channel state information (CSI), then it can adapt the transmit power level, symbol/bit rate, constellation size, coding rate/scheme or any combination of these parameters in response to the changing channel conditions [5]-[8]. In [6], the ergodic capacity of a two hop $\mathrm{AF}$ cooperative system with multiple relays and adaptive source transmission is explored; whereas, capacity of $\mathrm{AF}$ multi-hop relaying systems under adaptive transmission is studied in [8]. An upper bound of the outage probability of an AF based cooperative system with a single relay in Rician fading channels is derived in [9]. The capacity of adaptive transmission techniques in a two-hop AF cooperative system with Rician fading is studied in [10]; upper bounds of the capacity are derived in the form of infinite integrals by using moment generating function approach. However, all these works consider AF protocol; whereas, DF protocol is useful as it requires digital processing at relay contrary to

Corresponding author: Manav R. Bhatnagar is with Department of Electrical Engineering, Indian Institute of Technology Delhi, Hauz Khas, IN110016 New Delhi, India, email: manav@ee.iitd.ac.in the AF protocol. The capacity of adaptive modulation in a single relay based DF cooperative system over Rayleigh fading channels is studied in [11]. In this paper, by using a well known series representation of the modified Bessel function of the first kind, we use a probability density function (PDF) and cumulative distribution function (CDF) based approach which leads to power series expressions of the exact ergodic capacity of the adaptive transmission based DF system over dissimilar Rician fading channels. The proposed power series expressions converge for finite number of summation terms and are shown to be useful in giving significant insight of the ergodic capacity of the adaptive transmission techniques in the DF cooperative system over dissimilar Rician fading channels.

Our main contributions are as follows. i) We derive the PDF and CDF of minimum of two non-central Chi-square random variables (RVs) with two degrees of freedom in terms of converging power series. ii) The exact average capacity of a two-hop DF cooperative system with adaptive modulation over dissimilar Rician channels is derived by using these PDF and CDF expressions. iii) Different adaptive transmission techniques are compared on the basis of these expressions over DF Rician channels.

\section{Characterization of Minimum of Two}

Non-CEnTRAL ChI-SQuare Random Variables With

$$
\text { Two DEGREES OF FREEDOM }
$$

Let $G_{i} \sim \mathcal{N}\left(m_{i}, \sigma^{2} / 2\right), i=1,2$ be the two Normal distributed RVs with $m_{i}$ means and $\sigma^{2} / 2$ variance, then $U=G_{1}^{2}+G_{2}^{2} \sim \mathcal{X}_{2}(K, \bar{\gamma})$, where $K=s^{2} / \sigma^{2}, \bar{\gamma}=s^{2}+\sigma^{2}$, and $s^{2}=m_{1}^{2}+m_{2}^{2}$, will be non-central Chi-square distributed with two degrees of freedom as [12, Eq. (2.16)]

$$
f_{U}(u)=A e^{-a u} I_{0}(2 \sqrt{K a u}),
$$

where $f_{U}(u)$ denotes the PDF of $U, a=(1+K) / \bar{\gamma}, A=$ $a e^{-K}$, and $I_{0}(\cdot)$ denotes the modified Bessel function of the first kind of order zero [13]. Let us now define the following RV:

$$
\gamma_{z} \triangleq \min \left\{\gamma_{x}, \gamma_{y}\right\}
$$

where $\gamma_{x} \sim \mathcal{X}_{2}\left(K_{x}, \bar{\gamma}_{x}\right)$ and $\gamma_{y} \sim \mathcal{X}_{2}\left(K_{y}, \bar{\gamma}_{y}\right)$ be independent and non-identically distributed (i.n.i.d.) non-central Chisquare RVs with two degrees of freedom. The CDF and PDF of $\gamma_{z}$ are obtained in the following lemmas which are proved in Appendix A

Lemma 1: The CDF of $\gamma_{z}$ is given as

$$
\begin{aligned}
F_{\gamma_{z}}(\gamma)=1-A_{x} A_{y} \sum_{k=0}^{\infty} \sum_{n=0}^{k} \tilde{B}_{x}(n) \tilde{B}_{y}(k-n) \Gamma\left(n+1, a_{x} \gamma\right) \\
\times \Gamma\left(k-n+1, a_{y} \gamma\right),
\end{aligned}
$$

where $a_{x}=\left(1+K_{x}\right) / \bar{\gamma}_{x}, a_{y}=\left(1+K_{y}\right) / \bar{\gamma}_{y}, A_{x}=a_{x} e^{-K_{x}}$, $A_{y}=a_{y} e^{-K_{y}}, \tilde{B}_{x}(k)=B_{x}(k) / a_{x}^{k+1}, B_{y}(k)=B_{y}(k) / a_{y}^{k+1}$, 


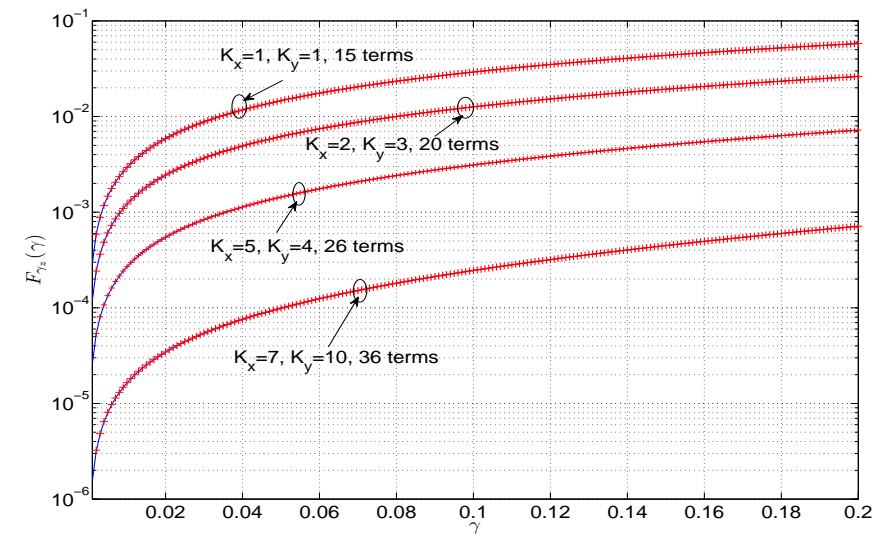

Fig. 1. Analytical + and simulated $-\mathrm{CDF}$ of $\gamma_{z}$ for different values of $K_{x}, K_{y}$, different number of summation terms, and $\bar{\gamma}_{x}=\bar{\gamma}_{y}=5$.

$B_{x}(k)=\frac{K_{x}^{k}\left(1+K_{x}\right)^{k}}{\bar{\gamma}_{x}^{k}\left(k !^{2}\right)}, B_{y}(k)=\frac{K_{y}^{k}\left(1+K_{y}\right)^{k}}{\bar{\gamma}_{y}^{k}\left(k !^{2}\right)}$, and $\Gamma(\cdot)$ denotes the Gamma function.

Lemma 2: The PDF of $\gamma_{z}$ is given as

$$
\begin{aligned}
f_{\gamma_{z}}(z)= & A_{x} A_{y} \sum_{k=0}^{\infty} \sum_{n=0}^{k} \tilde{B}_{x}(n) \tilde{B}_{y}(k-n)\left(a_{x}^{n+1}(k-n) !\right. \\
& \times \sum_{m_{1}=0}^{k-n} \frac{a_{y}^{m_{1}}}{m_{1} !} \gamma^{n+m_{1}} e^{-\left(a_{x}+a_{y}\right) \gamma}+a_{y}^{k-n+1} n ! \\
& \left.\times \sum_{m_{2}=0}^{n} \frac{a_{x}^{m_{2}}}{m_{2} !} \gamma^{k-n+m_{2}} e^{-\left(a_{x}+a_{y}\right) \gamma}\right) .
\end{aligned}
$$

It can be easily shown analytically that (3) and (4) contain converging power series. This fact is corroborated by Fig. 1 where the CDF of $\gamma_{z}$ given in (3) converges nicely to the simulated CDF for finite number of summation terms.

\section{System Model And CAPACITy AnAlysis Under ADAPTIVE TRANSMISSION}

Let us consider a DF cooperative system with a single halfduplex relay and no direct link in between the source and the destination. The channels of the source-to-relay and relay-todestination links are assumed to be i.n.i.d. Rician distributed with $K_{x}$ and $K_{y}$ line of sight (LOS) components, and average SNRs $\bar{\gamma}_{x}$ and $\bar{\gamma}_{y}$, respectively. Therefore, instantaneous SNRs of the source-to-relay and relay-to-destination links, i.e., $\gamma_{x} \sim \mathcal{X}_{2}\left(K_{x}, \bar{\gamma}_{x}\right)$ and $\gamma_{y} \sim \mathcal{X}_{2}\left(K_{y}, \bar{\gamma}_{y}\right)$, respectively, are non-central Chi-square RVs with two degrees of freedom.

The communication from the source to the destination takes place in two orthogonal channels via the relay that always decodes and forwards the data of the source to the destination. Hence, both hops act independently in the sense that decoding/encoding takes place at the intermediate relay. Consequently, the DF based cooperative transmission is equivalent to a series network, which means that the capacity of the system is dominated by the worst hop. Since the capacity is a monotonous function of SNR, the minimum of the capacities of the source-to-relay and relay-to-destination channels equals the capacity of the weakest of the source-to-relay and relayto-destination channels. Therefore, the equivalent SNR (not end-to-end SNR) of the two-hop DF system from a capacity point of view is given in (2) [11]. In the following subsections, we will derive the capacity of different adaptive schemes in DF cooperative system with Rician fading channels.

\section{A. Optimal Rate Adaptation with Constant Transmit Power}

For optimal rate adaptation to the fading level and constant transmit power, the channel capacity of the considered DF relaying system in bits per second is given by [5]

$$
C=0.5 B \int_{0}^{\infty} \log _{2}(1+\gamma) f_{\gamma_{z}}(\gamma) d \gamma
$$

where $B$ (in hertz) is the bandwidth of the channel. Let us now state the following lemma whose proof is given in Appendix B.

Lemma 3: For $\alpha \in \mathbb{Z}^{*}$ and $\beta>0$, we have

$$
\begin{aligned}
& \int_{0}^{\infty} w^{\alpha} e^{-\beta w} \ln (1+w) d w \\
& =\sum_{q=0}^{\alpha}{ }^{\alpha} C_{q}(-1)^{\alpha-q} \frac{e^{\beta}}{\beta^{q}} G_{2,3}^{3,0}\left(\beta \mid \begin{array}{c}
0,0 \\
-1,-1, q
\end{array}\right),
\end{aligned}
$$

where $G_{p, q}^{m, n}\left(v \mid \begin{array}{c}a_{1}, . ., a_{p} \\ b_{1}, . ., b_{q}\end{array}\right)$ is the Meijer's function, $\ln (\cdot)$ denotes the natural logarithm, and ${ }^{\mu} C_{\nu}$ denotes the binomial coefficient.

By substituting $f_{\gamma_{z}}(\gamma)$ from (4) in (5) and then using Lemma 3, we get the capacity of the DF relating with rate adaptation as follows:

$$
\begin{aligned}
& C=0.5 B A_{x} A_{y} \log _{2} e \sum_{k=0}^{\infty} \sum_{n=0}^{k} \tilde{B}_{x}(n) \tilde{B}_{y}(k-n)\left(a_{x}^{n+1}(k-n) !\right. \\
& \times \sum_{m_{1}=0}^{k-n} \frac{a_{y}^{m_{1}}}{m_{1} !} \sum_{q_{1}=0}^{n+m_{1}} n+m_{1} C_{q_{1}}(-1)^{n+m_{1}-q_{1}} \frac{e^{a_{x}+a_{y}}}{\left(a_{x}+a_{y}\right)^{q_{1}}} \\
& \times G_{2,3}^{3,0}\left(a_{x}+a_{y} \mid \begin{array}{c}
0,0 \\
-1,-1, q_{1}
\end{array}\right)+a_{y}^{k-n+1} n ! \sum_{m_{2}=0}^{n} \frac{a_{x}^{m_{2}}}{m_{2} !} \\
& \times \sum_{q_{2}=0}^{k-n+m_{2}} k-n+m_{2} C_{q_{2}}(-1)^{k-n+m_{2}-q_{2}} \frac{e^{a_{x}+a_{y}}}{\left(a_{x}+a_{y}\right)^{q_{2}}} \\
& \left.\times G_{2,3}^{3,0}\left(a_{x}+a_{y} \mid \begin{array}{c}
0,0 \\
-1,-1, q_{2}
\end{array}\right)\right) .
\end{aligned}
$$

\section{B. Optimal Simultaneous Power and Rate Adaptation}

Given an average transmit power constraint, the channel capacity of a fading channel with optimal power and rate adaptation is given as [5]

$$
C=0.5 B \int_{\gamma_{0}}^{\infty} \log _{2}\left(\frac{\gamma}{\gamma_{0}}\right) f_{\gamma_{z}}(\gamma) d \gamma
$$

where $\gamma_{0}$ is the optimal cutoff SNR level below which the data transmission is suspended. This optimal cutoff SNR must satisfy the following relation:

$$
\int_{\gamma_{0}}^{\infty}\left(\frac{1}{\gamma_{0}}-\frac{1}{\gamma}\right) f_{\gamma_{z}}(\gamma) d \gamma=1
$$

Let us now state the following lemma whose proof is given in Appendix C

Lemma 4: For $\alpha \geq 0, \beta>0$, and $\eta>0$, we have

$$
\int_{\eta}^{\infty} w^{\alpha} e^{-\beta w} \ln \left(\frac{w}{\eta}\right) d w=\frac{\eta}{\beta^{\alpha}} G_{2,3}^{3,0}\left(\beta \eta \mid \begin{array}{c}
0,0 \\
-1,-1, \alpha
\end{array}\right) .
$$

By substituting $f_{\gamma_{z}}(\gamma)$ from (4) in (8) and then using Lemma 4] we get the capacity of DF system with transmit 


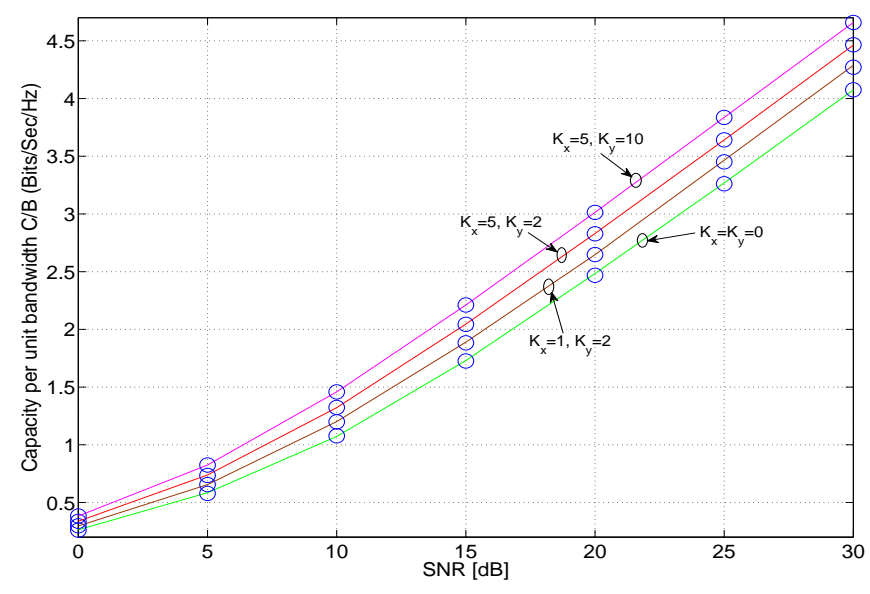

Fig. 2. Analytical - and simulated $\circ$ capacity of the DF system with adaptive rate and constant power for different values of $K_{x}, K_{y}$, and $\bar{\gamma}_{x}=$ $\bar{\gamma}_{y}=\bar{\gamma}$.

power and rate adaptation over dissimilar Rician fading hops as

$$
\begin{aligned}
C & =0.5 B A_{x} A_{y} \log _{2} e \sum_{k=0}^{\infty} \sum_{n=0}^{k} \tilde{B}_{x}(n) \tilde{B}_{y}(k-n)\left(a_{x}^{n+1}(k-n) !\right. \\
\times & \sum_{m_{1}=0}^{k-n} \frac{a_{y}^{m_{1}} \gamma_{0}}{m_{1} !\left(a_{x}+a_{y}\right)^{n+m_{1}}} \\
& \times G_{2,3}^{3,0}\left(\left(a_{x}+a_{y}\right) \gamma_{0} \mid-1,-1, n+m_{1}\right)+a_{y}^{k-n+1} n ! \\
& \times \sum_{m_{2}=0}^{n} \frac{a_{x}^{m_{2}} \gamma_{0}}{m_{2} !\left(a_{x}+a_{y}\right)^{k-n+m_{2}}} \\
& \left.\times G_{2,3}^{3,0}\left(\left(a_{x}+a_{y}\right) \gamma_{0} \mid-1,-1, k-n+m_{2}\right)\right) .
\end{aligned}
$$

The optimal cutoff SNR $\gamma_{0}$ is found by solving for $\gamma_{0}$ in (9), which can be rewritten as

$$
\frac{1}{\gamma_{0}} \int_{\gamma_{0}}^{\infty} f_{\gamma_{z}}(\gamma) d \gamma-\int_{\gamma_{0}}^{\infty} \frac{1}{\gamma} f_{\gamma_{z}}(\gamma) d \gamma=1
$$

Since $\int_{\gamma_{0}}^{\infty} f_{\gamma_{z}}(\gamma) d \gamma=1-F_{\gamma_{z}}\left(\gamma_{0}\right)$, from (3), we get

$$
\begin{gathered}
\frac{1}{\gamma_{0}} \int_{\gamma_{0}}^{\infty} f_{\gamma_{z}}(\gamma) d \gamma=\frac{A_{x} A_{y}}{\gamma_{0}} \sum_{k=0}^{\infty} \sum_{n=0}^{k} \tilde{B}_{x}(n) \tilde{B}_{y}(k-n) \\
\times \Gamma\left(n+1, a_{x} \gamma_{0}\right) \Gamma\left(k-n+1, a_{y} \gamma_{0}\right) .
\end{gathered}
$$

Further, it can be shown by using (4) and after some algebra that

$$
\begin{aligned}
& \int_{\gamma_{0}}^{\infty} \frac{1}{\gamma} f_{\gamma_{z}}(\gamma) d \gamma=A_{x} A_{y} \sum_{k=0}^{\infty} \sum_{n=0}^{k} \tilde{B}_{x}(n) \tilde{B}_{y}(k-n) \\
& \times\left(a_{x}^{n+1}(k-n) ! \sum_{m_{1}=0}^{k-n} \frac{a_{y}^{m_{1}}}{m_{1} !} \mathcal{G}\left(n+m_{1},\left(a_{x}+a_{y}\right) \gamma_{0}\right)\right. \\
& \left.+a_{y}^{k-n+1} n ! \sum_{m_{2}=0}^{n} \frac{a_{x}^{m_{2}}}{m_{2} !} \mathcal{G}\left(k-n+m_{2},\left(a_{x}+a_{y}\right) \gamma_{0}\right)\right)
\end{aligned}
$$

where

$$
\mathcal{G}\left(u, v \gamma_{0}\right)= \begin{cases}\frac{\Gamma\left(u, v \gamma_{0}\right)}{v^{u}}, & \text { if } u>0 \\ E_{1}\left(v \gamma_{0}\right), & \text { if } u=0\end{cases}
$$

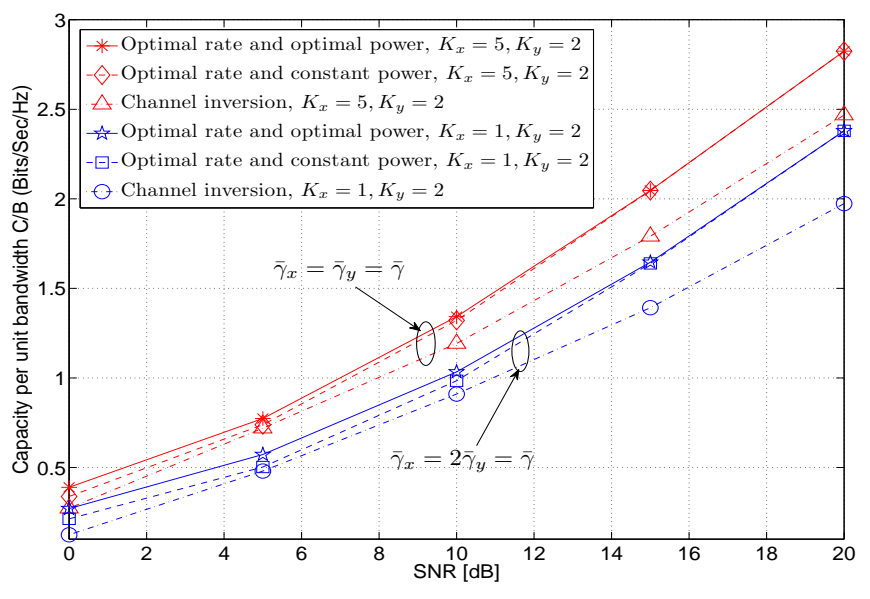

Fig. 3. Comparison of the capacity of the DF system under different adaptive modulation schemes and with different values of $K_{x}$ and $K_{y}$.

where $\Gamma(q, w)=\int_{w}^{\infty} t^{q-1} e^{-t} d t$ denotes the incomplete Gamma function and $E_{1}(w)=\int_{1}^{\infty} t^{-1} e^{-w t} d t, w \geq 0$ is the exponential integral. Substituting (13) and (14) into (9), the optimal cutoff SNR $\gamma_{0}$ can be obtained numerically by using MATLAB or MATHEMATICA. Since $\int_{\gamma_{0}}^{\infty} f_{\gamma_{z}}(\gamma) d \gamma \leq 1$ and $\int_{\gamma_{0}}^{\infty}\left(f_{\gamma_{z}}(\gamma) / \gamma\right) d \gamma \geq 0$, it can be seen from (12) that $0 \leq \gamma_{0} \leq 1$.

\section{Channel Inversion with Fixed Rate}

A low complexity adaptive transmission technique is truncated channel inversion with fixed rate where the transmitter only adjusts its power to maintain a constant SNR at the destination. Truncated channel inversion is applied if the SNR is above a cutoff $\beta_{0}$. The channel capacity in this case is given as [5]

$$
C=0.5 B \log _{2}\left(1+\frac{1}{\int_{\beta_{0}}^{\infty} \frac{f_{\gamma_{z}}(\gamma)}{\gamma} d \gamma}\right)\left(1-P_{\text {out }}\right),
$$

where $P_{\text {out }}$ denotes the probability of outage given by

$$
P_{\text {out }}=\operatorname{Pr}\left(\gamma<\beta_{0}\right)=\int_{0}^{\beta_{0}} f_{\gamma_{z}}(\gamma) d \gamma=F_{\gamma_{z}}\left(\beta_{0}\right) .
$$

The capacity under the channel inversion based adaptive transmission technique can be obtained by using (3), (14), and (16).

\section{Numerical Results AND Discussion}

In Fig. 2, we have plotted simulated and analytical values of the capacity of the DF system under optimal power adaptation and constant transmit power for different values of $K_{x}$ and $K_{y}$ and $\bar{\gamma}_{x}=\bar{\gamma}_{y}=\bar{\gamma}$. It can be seen from Fig. 2 that the simulation results match the analytical values, obtained from (7), closely. The analytical capacity of the DF system with different values of $K_{x}$ and $K_{y} ; \bar{\gamma}_{x}=\bar{\gamma}_{y}=\bar{\gamma}$; and $\bar{\gamma}_{x}=2 \bar{\gamma}_{y}=\bar{\gamma}$ is plotted for all three adaptive modulation schemes (discussed in Section III) in Fig. 3. In the optimal simultaneous rate and power adaptation scheme high power levels are assigned for good channel conditions [14]; therefore, the transmit power levels are very likely to be (almost) constant in large SNR regimes. Hence, optimal simultaneous rate and power adaptation provides (almost) no improvement 
in the capacity over the optimal rate adaptation and constant transmit power at high SNR values; however, a capacity gain is seen from low to moderate SNR values in Fig. 3. Since the optimal rate adaptation with constant power only adapts its rate, the DF system under Rician fading can opt for this less complexity technique than the optimal rate and power adaptive technique at high SNR values. Further, the optimized capacity (with the following constraint: $\beta_{0} \leq 1$ ) of the DF Rician system with channel inversion based low complexity adaptive scheme is poorer than the other two schemes in general, as seen in Fig. 3. Since $0 \leq \gamma_{0} \leq 1$ and the probability of outage increases with increasing $\beta_{0}$ (as can be seen from (17)), the constraint $\beta_{0} \leq 1$ is used to limit the maximum outage probability of the DF system.

The average SNR is denoted by $\bar{\gamma}$ and shown at the $\mathrm{x}$-axis of Figs. 2 and 3.

\section{APPENDIX A}

\section{Proof of LEMMA 1 AND LEMMA 2}

The CDF of $\gamma_{z}$ can be obtained after some algebra as follows:

$$
F_{\gamma_{z}}(\gamma)=1-\int_{\gamma}^{\infty} f_{\gamma_{x}}(x) d x \int_{\gamma}^{\infty} f_{\gamma_{y}}(y) d y .
$$

From (1) and by using the series representation of the modified Bessel function [15, Eq. (2.3.32)], we can write the PDFs of $\gamma_{x}$ and $\gamma_{y}$ as

$$
\begin{aligned}
f_{\gamma_{x}}(x) & =A_{x} e^{-a_{x} x} \sum_{k=0}^{\infty} B_{x}(k) x^{k}, \\
f_{\gamma_{y}}(y) & =A_{y} e^{-a_{y} y} \sum_{k=0}^{\infty} B_{y}(k) y^{k} .
\end{aligned}
$$

From 19 and after some algebra, it can be shown that

$$
\begin{aligned}
& \int_{\gamma}^{\infty} f_{\gamma_{x}}(x) d x=A_{x} \sum_{k=0}^{\infty} \tilde{B}_{x}(k) \Gamma\left(k+1, a_{x} \gamma\right), \\
& \int_{\gamma}^{\infty} f_{\gamma_{y}}(y) d y=A_{y} \sum_{k=0}^{\infty} \tilde{B}_{y}(k) \Gamma\left(k+1, a_{y} \gamma\right) .
\end{aligned}
$$

By using (20) and [13, Eq. (0.316)] in (18), we get (3). After taking the derivative of $F_{\gamma_{z}}(\gamma)$ in (3) with respect to $\gamma$, we have

$$
\begin{aligned}
& f_{\gamma_{z}}(\gamma)=A_{x} A_{y} \sum_{k=0}^{\infty} \sum_{n=0}^{k} \tilde{B}_{x}(n) \tilde{B}_{y}(k-n)\left(a_{x}^{n+1} \gamma^{n} e^{-a_{x} \gamma}\right. \\
& \left.\times \Gamma\left(k-n+1, a_{y} \gamma\right)+a_{y}^{k-n+1} \gamma^{k-n} e^{-a_{y} \gamma} \Gamma\left(n+1, a_{x} \gamma\right)\right) .
\end{aligned}
$$

We can further simplify (21) by using the following relation: $\Gamma(r+1, b z)=e^{-b z} r ! \sum_{m=0}^{r} \frac{b^{m} z^{m}}{m !}$, where $r$ is a positive integer, as given in (4).

\section{APPENDiX B \\ PROOF OF LEMMA 3}

Let us denote the left hand side of $(6)$ as

$$
\mathcal{I}=\int_{0}^{\infty} w^{\alpha} e^{-\beta w} \ln (1+w) d w .
$$

By substituting $1+w=t$ in the right hand side (RHS) of (22), we get

$$
\mathcal{I}=\int_{1}^{\infty}(t-1)^{\alpha} e^{-\beta(t-1)} \ln t d t .
$$

The following relation is obtained by using [13, Eq. (1.111)] in (23):

$$
\mathcal{I}=\sum_{q=0}^{\alpha}{ }^{\alpha} C_{q}(-1)^{\alpha-q} e^{\beta} \int_{1}^{\infty} t^{f(q)-1} e^{-\beta t} \ln t d t,
$$

where $f(q)=q+1$. From [13, Eq. (4.358.1)] and (24), we have

$$
\mathcal{I}=\sum_{q=0}^{\alpha}{ }^{\alpha} C_{q}(-1)^{\alpha-q} e^{\beta} \frac{\delta}{\delta f(q)}\left(\beta^{-f(q)} \Gamma(f(q), \beta)\right) .
$$

By using the following relation:

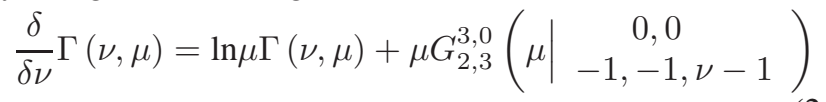

in (25) and after some algebra we get the RHS of (6).

\section{APPENDIX C \\ PRoOf of LeMma 4}

Lemma 4 can be proved by substituting $t=w / \eta$ in the left hand side of (10) and then using [13, Eq. (4.358.1)] and (26).

\section{REFERENCES}

[1] A. S. Avestimehr and D. N. C. Tse, "Outage capacity of the fading relay channel in the low-SNR regime," IEEE Trans. Inform. Theory, vol. 53, no. 4, pp. 1401-1415, Apr. 2007.

[2] N. C. Beaulieu and J. Hu, "A closed-form expression for the outage probability of decode-and-forward relaying in dissimilar Rayleigh fading channels," IEEE Commun. Lett., vol. 10, no. 12, pp. 813-815, Dec. 2006.

[3] H. A. Suraweera, P. J. Smith, and J. Armstrong, "Outage probability of cooperative relay networks in Nakagami- $m$ fading channels," IEEE Commun. Lett., vol. 10, no. 12, pp. 834-836, Dec. 2006.

[4] S. Shrestha and K.-H. Chang, "Analysis of outage capacity performance for cooperative $\mathrm{DF}$ and $\mathrm{AF}$ relaying in dissimilar Rayleigh fading channels," IEEE International Symposium on Information Theory, 2008 (ISIT 2008), pp. 494 -498, July 2008, Toronto, Canada.

[5] M.-S. Alouini and A. J. Goldsmith, "Capacity of Rayleigh fading channels under different adaptive transmission and diversity-combining techniques," IEEE Trans. Vehicular Technol., vol. 48, no. 4, pp. 11651181, July 1999.

[6] T. Nechiporenko, K. T. Phan, C. Tellambura, and H. H. Nguyen, "On the capacity of Rayleigh fading cooperative systems under adaptive transmission," IEEE Trans. Wireless Commun., vol. 8, no. 4, pp. 16261631, Apr. 2009.

[7] B. M. A. Annamalai and R. Palat, "Unified analysis of ergodic capacity of cooperative non-regenerative relaying with adaptive source transmission policies," in GLOBECOM Workshops (GC Wkshps-HeterWMN), pp. 175-180, Dec. 2010, Miami, Florida, USA.

[8] G. Farhadi and N. C. Beaulieu, "Capacity of amplify-and-forward multi-hop relaying systems under adaptive transmission," IEEE Trans. Commun., vol. 58, no. 3, pp. 758-763, Mar. 2010.

[9] W. Limpakom, Y.-D. Yao, and H. Man, "Outage probability analysis of wireless relay and cooperative networks in Rician fading channels with different $K$-factors," IEEE 69th Vehicular Technology Conference, 2009 (VTC Spring 2009), pp. 1-5, Apr. 2009, Hoboken, NJ, USA.

[10] B. Modi, O. Olabiyi, A. Annamalai, and D. Vaman, "On ergodic capacity of cooperative non-regenerative relay networks in Rice fading environments," IEEE GLOBECOM Workshops (GC Wkshps), 2011, pp. 348 -352, Dec. 2011, Houstan, Texas, USA.

[11] M. Hasna, "On the capacity of cooperative diversity systems with adaptive modulation," in Proc. Second IFIP International Conference on Wireless and Optical Communications Networks, 2005 (WOCN 2005), pp. 432 - 436, March 2005, Doha, Qatar.

[12] M. K. Simon and M.-S. Alouini, Digital Communication over Fading Channels. New Jersey, USA: John Wiley \& Sons, Inc., 2005.

[13] I. S. Gradshteyn and I. M. Ryzhik, Table of Integrals, Series, and Products, 7th ed., A. Jeffrey and D. Zwillinger, Eds. Burlington, MA, USA: Academic Press, 2007.

[14] M.-S. Alouini and A. Goldsmith, "Capacity of Rayleigh fading channels under different adaptive transmission and diversity-combining techniques," IEEE Trans. Vehicular Technol., vol. 48, no. 4, pp. 1165 1181, Jul. 1999.

[15] J. G. Proakis and M. Salehi, Digital Communications, 5th ed. New York, USA: McGraw-Hill Book Company, 2008. 